\title{
A reference linkage map for Eucalyptus
}

\author{
Corey J Hudson ${ }^{1 *}$, Jules S Freeman ${ }^{1,2}$, Anand RK Kullan ${ }^{3}$, César D Petroli ${ }^{4}$, Carolina P Sansaloni ${ }^{4}$, Andrzej Kilian ${ }^{5}$, \\ Frank Detering ${ }^{5}$, Dario Grattapaglia ${ }^{6}$, Brad M Potts ${ }^{1}$, Alexander A Myburg ${ }^{3}$ and René E Vaillancourt ${ }^{1}$
}

\begin{abstract}
Background: Genetic linkage maps are invaluable resources in plant research. They provide a key tool for many genetic applications including: mapping quantitative trait loci (QTL); comparative mapping; identifying unlinked (i.e. independent) DNA markers for fingerprinting, population genetics and phylogenetics; assisting genome sequence assembly; relating physical and recombination distances along the genome and map-based cloning of genes. Eucalypts are the dominant tree species in most Australian ecosystems and of economic importance globally as plantation trees. The genome sequence of $E$. grandis has recently been released providing unprecedented opportunities for genetic and genomic research in the genus. A robust reference linkage map containing sequence-based molecular markers is needed to capitalise on this resource. Several high density linkage maps have recently been constructed for the main commercial forestry species in the genus (E. grandis, E. urophylla and E. globulus) using sequenced Diversity Arrays Technology (DArT) and microsatellite markers. To provide a single reference linkage map for eucalypts a composite map was produced through the integration of data from seven independent mapping experiments (1950 individuals) using a marker-merging method.
\end{abstract}

Results: The composite map totalled 1107 cM and contained 4101 markers; comprising 3880 DArT, 213 microsatellite and eight candidate genes. Eighty-one DArT markers were mapped to two or more linkage groups, resulting in the 4101 markers being mapped to 4191 map positions. Approximately 13\% of DArT markers mapped to identical map positions, thus the composite map contained 3634 unique loci at an average interval of $0.31 \mathrm{cM}$.

Conclusion: The composite map represents the most saturated linkage map yet produced in Eucalyptus. As the majority of DArT markers contained on the map have been sequenced, the map provides a direct link to the E. grandis genome sequence and will serve as an important reference for progressing eucalypt research.

\section{Background}

Genetic linkage maps are valuable resources which can be used to provide a framework for many genomic analyses. Linkage maps can be used to investigate the organisation and evolution of genomes through comparative mapping [1-3] and serve as a basis for investigating phenotypic traits of ecological and economic importance through the localisation of quantitative trait loci [QTL; 4-6]. Subsequently, QTL results may be used to help guide the selection of candidate genes for association studies or be applied in marker-assisted breeding programmes $[7,8]$. Linkage maps can also be used to anchor physical maps and assist in the assembly of genome sequences [9-11]. The wide application of linkage maps in combination with

\footnotetext{
* Correspondence: cjhudson@utas.edu.au

'School of Plant Science and CRC for Forestry, University of Tasmania, Private Bag 55 Hobart, Tasmania 7001, Australia

Full list of author information is available at the end of the article
}

their value to genetics research has led to numerous linkage mapping projects being undertaken in plants. Detailed linkage maps have been produced for all of the world's staple cereal species [12], and in forest trees, linkage maps have been produced for many of the most widely-planted species due to their commercial importance as wood and fibre crops $[1,13,14]$.

Grattapaglia and Sederoff [15] published the first genetic linkage map in the forest tree genus Eucalyptus in 1994. Subsequently, many mapping pedigrees have been established for the purpose of linkage map construction and associated QTL analyses. More than 20 eucalypt genetic linkage maps have been reported with most being produced in the main commercially grown species, or their hybrids, from the Eucalyptus subgenus Symphyomyrtus. Thus, the majority of linkage mapping projects have focussed on E. grandis, E. urophylla and E. globulus [reviewed in 16], while a smaller number of maps have 
also been produced for E. nitens [17], E. teriticornis [18,19], E. camaldulensis [20] and for species in the closely related genus Corymbia [21].

Many early eucalypt linkage maps were constructed using random amplification of polymorphic DNA (RAPD) and amplified fragment length polymorphism (AFLP) molecular markers [16,22]. However, the anonymous nature of these dominant markers has limited the transfer of linkage information between studies $[16,23]$. More informative, codominant markers such as isozyme and random fragment length polymorphism (RFLPs) have also been used in eucalypt linkage mapping, although, their low throughput, low inter-pedigree polymorphism and labour intensive genotyping requirements have limited their use [16,23]. The more recent development of highly polymorphic microsatellite markers made available a large potential suite of markers that are transferrable between species and polymorphic in multiple pedigrees. This enabled linkage group synteny to be established between maps containing common microsatellite markers and the positions and stability of QTL across multiple species to be examined [e.g. 24-27]. The ability to establish linkage group synteny has also enabled moderate-density comparative mapping studies $[23,28]$.

Recent advances in molecular methods have led to high-throughput genotyping systems being developed [e.g. 29,30]. These have made it possible to quickly generate many hundreds of markers in single mapping pedigrees and have helped facilitate the construction of high density linkage maps [12]. Most recently in Eucalyptus, Diversity Arrays Technology [DArT; 31] has been used to generate large numbers of molecular markers for genetic linkage mapping in several mapping pedigrees [e.g. 11,32,33]. The eucalypt DArT markers are highly transferable across species from subgenus Symphyomyrtus [34] and the high-throughput array-based genotyping system provides wide genome coverage [35]. A key benefit of the Eucalyptus DArT markers is the public availability of the sequences of most of the 7680 markers contained on the genotyping array [GenBank accession numbers HR865291 - HR872186], thus making it possible to anchor DArT markers directly to the reference E. grandis genome sequence [v1.0 released January 2011; 36]. However, while the DArT technology offers many advantages, the DArT markers do suffer some limitations due to their dominant nature. For example, the incomplete segregation information provided by those DArT markers segregating in a 3:1 ratio (intercross) results in an exponential increase of marker-ordering calculations compared to fully-informative co-dominant markers [37]. Co-dominant markers also provide more complete information in QTL mapping studies [e.g. allowing estimation of additive and dominant allelic effects; 38] and are more useful in some genetic analyses, such as estimating population genetic parameters (e.g. inbreeding levels), relative to dominant marker types such as DArT. In addition, the DArT marker assay can be subject to cross-hybridization from duplicated loci in the genome, although most such artifacts can be excluded by preselecting markers exhibiting Mendelian segregation ratios in mapping pedigrees.

At present, DArT markers have been used to construct linkage maps in seven independent E. globulus and/or E. grandis $\times E$. urophylla hybrid family mapping pedigrees $[11,32,33]$. All of these maps also contain a variable number of co-dominant microsatellite markers, which provide important links to many earlier eucalypt linkage maps. In the two largest mapping pedigrees (more than 500 individuals each), 1010 [32] and 2229 [33] DArT markers, were mapped at sub-centiMorgan marker densities and collectively more than 4000 DArT and microsatellite markers have been mapped in the seven pedigrees.

All DArT marker based linkage maps were constructed using the program JoinMap 4.0 [37]. This program is one of the most commonly used linkage mapping programs and appears to be the only software available for building linkage maps using the combined segregation data from multiple populations [39-41]. However, it is presently not feasible to combine the segregation data contained within the seven eucalypt mapping families describe above (collectively 1950 individuals), and successfully order such large numbers of markers within linkage groups (up to $\sim 500$ ) due to computational limitations (Van Ooijen pers comm.). To circumvent the limitations of traditional segregation-based methods of linkage map construction, alternative marker-merging strategies have been developed. A so-called 'composite map' can be produced in which markers from individual component maps are merged into a single map based on their position relative to common anchor loci. For example, the 'neighbours' marker-merging approach of Cone et al. [42] and the marker-merging method implemented in the PhenoMap program (GeneFlow Inc. USA) have been used to successfully construct high density composite maps containing several thousand markers in a number of plant species; including Sorghum [43], barley $[41,44,45]$ and maize $[42,46]$.

In this study, a marker-merging method was used to construct a high-density DArT and microsatellite marker composite linkage map from seven independently constructed maps. Recent comparative mapping analyses using 236 to 393 markers shared between three of the maps [see 32] showed that these linkage maps exhibited high synteny ( $>93.4 \%$ markers occurring on the same linkage groups) and high colinearity ( $>93.7 \%$ markers having the same order within linkage groups). This indicated that it would be possible to merge markers from 
several component maps into a single high quality map featuring robust marker-order together with very high marker density. It is expected that this composite map will facilitate marker and map information exchange and serve as a valuable reference for species in the subgenus Symphyomyrtus.

\section{Methods}

The following terms are used to describe the various types of linkage maps reported in this paper; (1) sexaveraged map - a consensus of individually constructed male and female maps, built in a single family using segregation data from both parents, (2) consensus map - a consensus of multiple individually constructed male and female maps, built in multiple families (e.g. $F_{2}$ doublepseudo backcross) using segregation data from all of the families, and (3) composite map - an integrated map of multiple sex-averaged and/or consensus maps, built using a marker-merging method.

\section{Component maps}

The composite map was built using an E. grandis $\times$ E. urophylla $\mathrm{F}_{2}$ double pseudo-backcross pedigree consensus linkage map [both species from section Latoangulatae; 33 ] plus one E. grandis $\times E$. urophylla sex-averaged map constructed in a $\mathrm{F}_{1}$ hybrid pedigree [11] and five purespecies E. globulus [section Maidenaria; 32] sex-averaged linkage maps constructed in either outcrossed $F_{2}$ or $F_{1}$ families (hereafter referred to as 'component' maps). Component map family sizes ranged from $172\left(\right.$ GLOB-F $\left.{ }_{2}-1\right)$ to 547 (GU-SA) and collectively contained 1,950 individuals (Table 1). The component maps were constructed by different researchers. All used JoinMap 4.0 [37] with marker-ordering within linkage groups (LGs) estimated using the regression algorithm of Stam [47] combined with the Kosambi mapping function. All component maps comprised 11 linkage groups in accordance with the haploid chromosome number of Eucalyptus [48].

Before building the composite map, marker names were standardised across maps, homologous linkage groups were identified using common (anchor) loci and marker colinearity between component maps was visually inspected in MapChart [49]. Map data was supplied for both framework (1032-marker) and comprehensive (2484-marker) maps built in the GU-Emb family [see 11]. Based on the level of marker-order agreement between linkage groups from these maps with other component maps, either GUEmb framework (LG's 1, 3, 5, 7 and 9) or comprehensive (LG's 2, 4, 6, 8, 10 and 11) linkage groups were included in composite map construction. Five linkage groups from three of the smaller E. globulus mapping families (Table 1) were found to have substantial regions of non-colinearity (discordant marker-orders) with other component maps. Consequently, LG6 and LG10 from the GLOB-F $F_{1}-1$ map, LG4 and LG9 from the GLOB-F $F_{1}-4$ and LG4 from the GLOB-F $F_{1}-5$ map were excluded from composite map construction.

The number of markers included for composite map construction ranged from $498\left(\mathrm{GLOB}_{-} \mathrm{F}_{1}-4\right)$ to 2290 (GU-SA; Table 1). In total, this consisted of 4350 individual markers, including: 4089 DArT, 253 microsatellites and eight mapped genes. Ninety-six markers $(2.2 \%$ of the total number of markers; termed 'multicopy' markers) were mapped to two or more linkage groups across component maps. This resulted in the 4350 individual markers being mapped to 4457 positions. Of these 4457 positions, 1960 could be considered to be bridging loci, meaning that these markers had been mapped to syntenic linkage groups in two or more component maps and would serve as anchor loci during composite map construction. Conversely, 2497 marker positions were unique to single component maps.

Table 1 Component map details

\begin{tabular}{|c|c|c|c|c|c|c|c|c|}
\hline \multirow[t]{2}{*}{ Linkage map ${ }^{a}$} & \multirow[t]{2}{*}{$\begin{array}{c}\text { Map } \\
\text { abbreviation }\end{array}$} & \multirow[t]{2}{*}{$n$} & \multirow[t]{2}{*}{ cM } & \multirow[t]{2}{*}{ MMI } & \multicolumn{4}{|c|}{$\begin{array}{c}\text { Markers mapped } \\
\text { (percentage of unique markers in pedigree) }\end{array}$} \\
\hline & & & & & DArT & SSR & Gene & Total \\
\hline E. grandis $\times$ E. urophylla SA double pseudo-backcross $F_{2}{ }^{b}$ & GU-SA & 547 & 1107 & 0.51 & $2229(45 \%)$ & $59(46 \%)$ & $2(100 \%)$ & $2290(45 \%)$ \\
\hline E. grandis $\times$ E. urophylla Embrapa $\mathrm{F}_{1}$ ce & GU-Emb & 177 & 1229 & 0.78 & $1617(41 \%)$ & $193(77 \%)$ & 0 & $1810(44 \%)$ \\
\hline E. globulus Lighthouse $F_{2}{ }^{d}$ & GLOB-LH & 503 & 1151 & 1.21 & $1010(27 \%)$ & $50(12 \%)$ & 0 & $1060(27 \%)$ \\
\hline E. globulus FAM1 $\mathrm{F}_{1}{ }^{\mathrm{d}}$ & $\mathrm{GLOB}_{-} \mathrm{F}_{1}-1$ & 184 & 1033 & 1.97 & $571(14 \%)$ & $4(0 \%)$ & $2(0 \%)$ & $577(14 \%)$ \\
\hline E. globulus FAM4 $\mathrm{F}_{1}{ }^{\mathrm{d}}$ & GLOB-F ${ }_{1}-4$ & 184 & 1137 & 2.46 & $488(10 \%)$ & $6(0 \%)$ & $4(25 \%)$ & $498(10 \%)$ \\
\hline E. globulus FAM5 $\mathrm{F}_{1}{ }^{\mathrm{d}}$ & GLOB-F ${ }_{1}-5$ & 183 & 1055 & 2.09 & $600(22 \%)$ & $4(0 \%)$ & $2(0 \%)$ & $606(21 \%)$ \\
\hline E. globulus FAM1 $\mathrm{F}_{2}{ }^{\mathrm{d}}$ & $\mathrm{GLOB}-\mathrm{F}_{2}-1$ & 172 & 1258 & 2.73 & $660(18 \%)$ & $30(30 \%)$ & $5(40 \%)$ & $695(18 \%)$ \\
\hline
\end{tabular}

Summary of the component maps used to construct the composite map. For each map, progeny size ( $n$ ), map length (cM; total for all 11 linkage groups), mean marker interval (MMl; average for all 11 linkage groups) and total number of mapped markers (using only those linkage groups included in composite map construction; see Methods) are given. For DArT, microsatellite (SSR) and gene markers mapped on each component map, the percentage of markers unique to that map (i.e. not mapped in any of the six other component maps) are given in parentheses. ${ }^{a}$ Cross details and reference; ${ }^{b}$ Kullan et al. [33], ${ }^{\mathrm{C}}$ Petroli et al. [11] and ${ }^{\mathrm{d}}$ Hudson et al. [32]. ${ }^{\mathrm{e}}$ Data for the E. grandis $\times$ E. urophylla Embrapa $\mathrm{F}_{2}$ component map calculated using a combination of framework and comprehensive linkage groups (see Methods). 


\section{Composite map construction}

The composite linkage map was constructed at Diversity Arrays Technology (DArT) Pty Ltd (Canberra, Australia) using specially developed $\mathrm{R}$ scripts which merged component map markers into the composite map based on their relative map positions. The $E$. grandis $\times E$. urophylla $\mathrm{SA}_{2} \quad \mathrm{~F}_{2}$ (GU-SA) linkage map was used as the seed-map (i.e. the 'fixed backbone' to which markers from other component maps were added) due to it having the largest progeny size, the largest number of both mapped and unique markers (Table 1) and high overall marker colinearity to the 11 main superscaffolds of the assembled E. grandis genome sequence $[33,36]$. The procedure for building each composite map linkage group was as follows. Firstly, the number of common markers in each seedmap - component map linkage group comparison was identified. Spearman rank marker-order correlations were then estimated and a heuristic 'fit value' for each comparison was calculated as; Fit value $=$ correlation $\times \log$ (number of common markers); where the second term rewards for the number of common markers with a diminishing returns function. Following selection of the component map linkage group with the highest Fit value, unique markers (i.e. those not mapped on the seed linkage group, or the "building' composite linkage group in following rounds) were added to the seed linkage group (or 'building' composite map linkage group) using linear regression. Here, the slope $(\mathrm{m})$ and intersect $(\mathrm{c})$ calculated from fitting the positions of common markers on the seed linkage group (pc) to their positions on the selected component map (pi) linkage group $(\mathrm{pc}=\mathrm{m} \times \mathrm{pi}+\mathrm{c})$ was used to calculate the positions of unique component map markers added to the seed linkage group. Once this first round was completed, the remaining component linkage groups were compared to this new 'building' composite map linkage group and the process was repeated. This continued until all unique markers had been added from remaining component maps which shared at least three common markers with the building composite map linkage group and had a marker-order correlation coefficient $\geq 0.50$. This process was repeated for each linkage group to yield the final composite map of 11 linkage groups. Markers which mapped to the distal ends of composite linkage groups and which had relatively large intermarker intervals $(\geq 5 \mathrm{cM}$ ) and poor support (e.g. mapped in one component map only) were removed. The numbering and orientation of linkage groups followed the convention established in Brondani et al. [23]; this also corresponds to the numbering of pseudochromosome assemblies in the E. grandis genome sequence [36].

\section{Composite map features}

Following composite map construction, marker-order correlations between composite and component map linkage groups were calculated in SAS 9.2 (SAS Institute, Cary, USA) using the PROC CORR Spearman function. To test whether multicopy markers were distributed equally across linkage groups, a $\chi^{2}$ test was used to compare the observed versus expected number of multicopy marker positions occurring on each linkage group. The expected number of multicopy markers per linkage group was calculated as; (total number of multicopy marker positions in the composite map/total number of DArT marker positions in the composite map) $\times$ number of DArT marker positions per linkage group for that linkage group. The BLAST server available at Phytozome [36] was used to search for DArT marker duplications. The bl2seq tool at NCBI [50] was used to examine DArT marker sequence similarity/redundancy. All graphical representations of linkage maps were drawn using MapChart [49].

\section{Results}

\section{Composite map details}

A total of 4101 individual markers, comprising 3880 DArT markers, eight gene-based markers and 213 microsatellite markers were included in the composite map. The composite map totalled $1107 \mathrm{cM}$ which was within the range of component map lengths (1033$1258 \mathrm{cM}$; Table 1) and contained only eleven marker intervals $\geq 3 \mathrm{cM}$; with a maximum marker interval of $5.9 \mathrm{cM}$. The composite map contained 81 multicopy DArT markers (2.1\% of total DArT markers) which were mapped to 171 map positions. Most multicopy markers occurred on two linkage groups only, however, one marker (ePt-574238) mapped to three linkage groups while four markers (ePt-503174, ePt-568818, ePt-637610, ePt637861) mapped to four linkage groups. This resulted in the 4101 markers being mapped to 4191 positions (Table 2). Over half (2171 or 53\%) of the markers mapped to these 4191 map positions had been mapped in a single component map only (i.e. were not shared among multiple component maps). Approximately 13\% of DArT markers mapped to identical positions in the composite map. Therefore, the map contained 3634 unique map loci with an average interval of $0.31 \mathrm{cM}$.

The number of multicopy DArT marker positions on each linkage group ranged from 5 to 24 and represented 1.9-6.4\% of the total number of DArT markers mapped per linkage group (Table 2). Although LG5 and LG7 contained a larger proportion of multicopy DArT marker positions (e.g. LG1 contained only 5 multicopy DArT marker positions, or $1.9 \%$ of the total number of DArT marker positions; Table 3), the proportion of multicopy DArT marker positions found on each linkage 
Table 2 Composite map summary

\begin{tabular}{|c|c|c|c|c|c|c|c|}
\hline \multirow[t]{2}{*}{ LG } & \multirow[t]{2}{*}{ cM } & \multicolumn{4}{|c|}{ Markers mapped } & \multirow{2}{*}{$\begin{array}{c}\text { Average } \\
\text { marker } \\
\text { interval } \\
\text { (cM) }\end{array}$} & \multirow{2}{*}{$\begin{array}{c}\text { MC } \\
\text { DArT } \\
\text { pos. }^{\text {a }}\end{array}$} \\
\hline & & DArT & SSR & Genes & Total & & \\
\hline 1 & 93.8 & 250 & 12 & 0 & 262 & 0.42 & 5 \\
\hline 2 & 102.1 & 451 & 29 & 0 & 480 & 0.24 & 18 \\
\hline 3 & 105.6 & 429 & 18 & 2 & 449 & 0.28 & 21 \\
\hline 4 & 80.9 & 219 & 9 & 3 & 231 & 0.41 & 12 \\
\hline 5 & 95.9 & 366 & 8 & 0 & 374 & 0.30 & 24 \\
\hline 6 & 125.3 & 408 & 43 & 1 & 452 & 0.31 & 15 \\
\hline 7 & 87.7 & 305 & 9 & 1 & 315 & 0.33 & 18 \\
\hline 8 & 137.3 & 540 & 26 & 0 & 566 & 0.28 & 19 \\
\hline 9 & 82.9 & 312 & 20 & 0 & 332 & 0.29 & 10 \\
\hline 10 & 97.8 & 336 & 20 & 1 & 357 & 0.30 & 12 \\
\hline 11 & 97.3 & 354 & 19 & 0 & 373 & 0.31 & 17 \\
\hline Total & 1106.5 & 3970 & 213 & 8 & 4191 & 0.31 & $171^{\mathrm{b}}$ \\
\hline
\end{tabular}

Summary of the composite map: including the number of mapped markers, length and average marker intervals by linkage group (LG). ${ }^{\mathrm{a}} \mathrm{MC}$ DArT pos. indicates the number of multicopy (MC) DArT marker positions (pos.) occurring on each linkage group. ${ }^{\mathrm{b}}$ The 171 multicopy DArT marker positions represent 81 multicopy DArT markers (see Additional file 1).

group did not significantly differ from that expected by chance across all linkage groups $\left(\chi^{2}=12.99, P=0.22\right.$, $d f=10)$. There was no trend within linkage groups for multicopy DArT markers to be clumped in either distal or central linkage group areas (data not shown). Composite map marker details, component map(s) marker origins and multicopy DArT marker information is presented in Additional file 1.

\section{Composite - component map colinearity}

Colinearity between component and composite map linkage groups can be viewed graphically in Figure 1 (for the GLOB-LH map) and in Additional file 2 (all component maps). Pair-wise linkage group markerorder correlations were generally high (greater than 0.90; Table 3) reflecting the high colinearity shown between common markers (Figure 1 and Additional file 2). However, a small degree of non-colinearity did occur between all component maps and the composite map. Eleven component map linkage groups had marker-order correlations of less than 0.90 (Table 3), however, these linkage groups were either, (1) identified as having poor marker colinearity with other component maps prior to composite map construction and excluded from analysis (five linkage groups with gray shading in Table 3), or (2) markerorder information from these linkage groups was not incorporated during composite map construction (correlation value without asterisk; six linkage groups Table 3) due to markers from these maps being previously added from other linkage groups having better fit values. Thus, these poorly correlated linkage groups did not adversely affect the composite map marker-order. For each linkage group, the average pair-wise marker-order correlation between the

Table 3 Composite - component map marker-order correlation coefficients

\begin{tabular}{|c|c|c|c|c|c|c|c|c|}
\hline \multirow[t]{2}{*}{ Composite map LG } & \multicolumn{8}{|c|}{ Component map } \\
\hline & $\overline{\text { GLOB-LH }}$ & GU-Emb & GLOB-F $F_{2}-1$ & GLOB-F $_{1}-1$ & GLOB-F $F_{1}-4$ & GLOB-F $_{1}-5$ & Average $^{a}$ & Average $^{\mathrm{b}}$ \\
\hline 1 & $0.98^{*}$ & $0.56^{\mathrm{F}}$ & $0.99^{*}$ & $0.99^{*}$ & $0.98^{*}$ & $0.95^{*}$ & 0.91 & 0.98 \\
\hline 2 & $0.98^{*}$ & $0.95^{\mathrm{C} *}$ & $0.93^{*}$ & $0.95^{*}$ & $0.98^{*}$ & 0.85 & 0.94 & 0.96 \\
\hline 3 & $0.91^{*}$ & $0.99^{\mathrm{F} *}$ & $0.97^{*}$ & $0.99^{*}$ & $0.97^{*}$ & $0.99^{*}$ & 0.97 & 0.97 \\
\hline 4 & $0.98^{*}$ & $0.74^{C}$ & $0.96^{*}$ & 0.89 & $0.79^{\mathrm{ex}}$ & $0.19^{\mathrm{ns}, \mathrm{ex}}$ & 0.76 & 0.97 \\
\hline 5 & $0.99^{*}$ & $0.92^{F}$ & $0.96^{*}$ & $0.96^{*}$ & $0.99^{*}$ & $0.96^{*}$ & 0.96 & 0.97 \\
\hline 6 & $0.99^{*}$ & $0.99^{C_{*}}$ & $0.99^{*}$ & $0.63^{\mathrm{ex}}$ & $0.99^{*}$ & 0.86 & 0.91 & 0.99 \\
\hline 7 & $0.98^{*}$ & $0.65^{\mathrm{F}}$ & $0.99^{*}$ & $0.98^{*}$ & $0.96^{*}$ & $0.91^{*}$ & 0.91 & 0.96 \\
\hline 8 & $0.98^{*}$ & $0.99^{\mathrm{C} *}$ & $0.99^{*}$ & 0.66 & $0.94^{*}$ & $0.99^{*}$ & 0.93 & 0.98 \\
\hline 9 & $0.99^{*}$ & $0.97^{F_{*}}$ & $0.98^{*}$ & $0.97^{*}$ & $0.65^{e x}$ & 0.97 & 0.92 & 0.98 \\
\hline 10 & $0.95^{*}$ & $0.98^{\mathrm{C} *}$ & $0.97^{*}$ & $0.35^{\mathrm{ns}, \mathrm{ex}}$ & 0.92 & $0.97^{*}$ & 0.86 & 0.97 \\
\hline 11 & $0.99^{*}$ & $0.99^{C *}$ & $0.99^{*}$ & $0.97^{*}$ & 0.96 & $0.99^{*}$ & 0.98 & 0.99 \\
\hline Average $^{c}$ & 0.98 & 0.88 & 0.97 & 0.85 & 0.92 & 0.87 & & \\
\hline Average $^{d}$ & 0.98 & 0.98 & 0.97 & 0.97 & 0.97 & 0.96 & & \\
\hline
\end{tabular}

Marker-order correlations between composite map and component map linkage groups; the GU-SA component map is not shown as this map was used as the seed-map (i.e. provided a fixed-order and all correlations were 1.0). For the GU-Emb component map, superscript letters indicates whether the framework (F) or comprehensive (C) linkage group was used in map construction. Component map linkage groups initially excluded from composite map construction are indicated by ${ }^{\text {ex }}$ superscript. An asterisk following the correlation value indicates that marker-order information from the component map was incorporated during construction of the composite map linkage group. Apart from two correlations (indicated by ${ }^{\mathrm{ns}}$ superscript) all correlations were significant at a $\leq 0.05$. Averages: ${ }^{\mathrm{a}}$ calculated using all six component maps, ${ }^{\mathrm{b}}$ calculated using only those linkage groups included in composite map construction (marked with an asterisk), c calculated using all 11 linkage groups, ${ }^{d}$ calculated using only those linkage groups included in composite map construction (marked with an asterisk). 


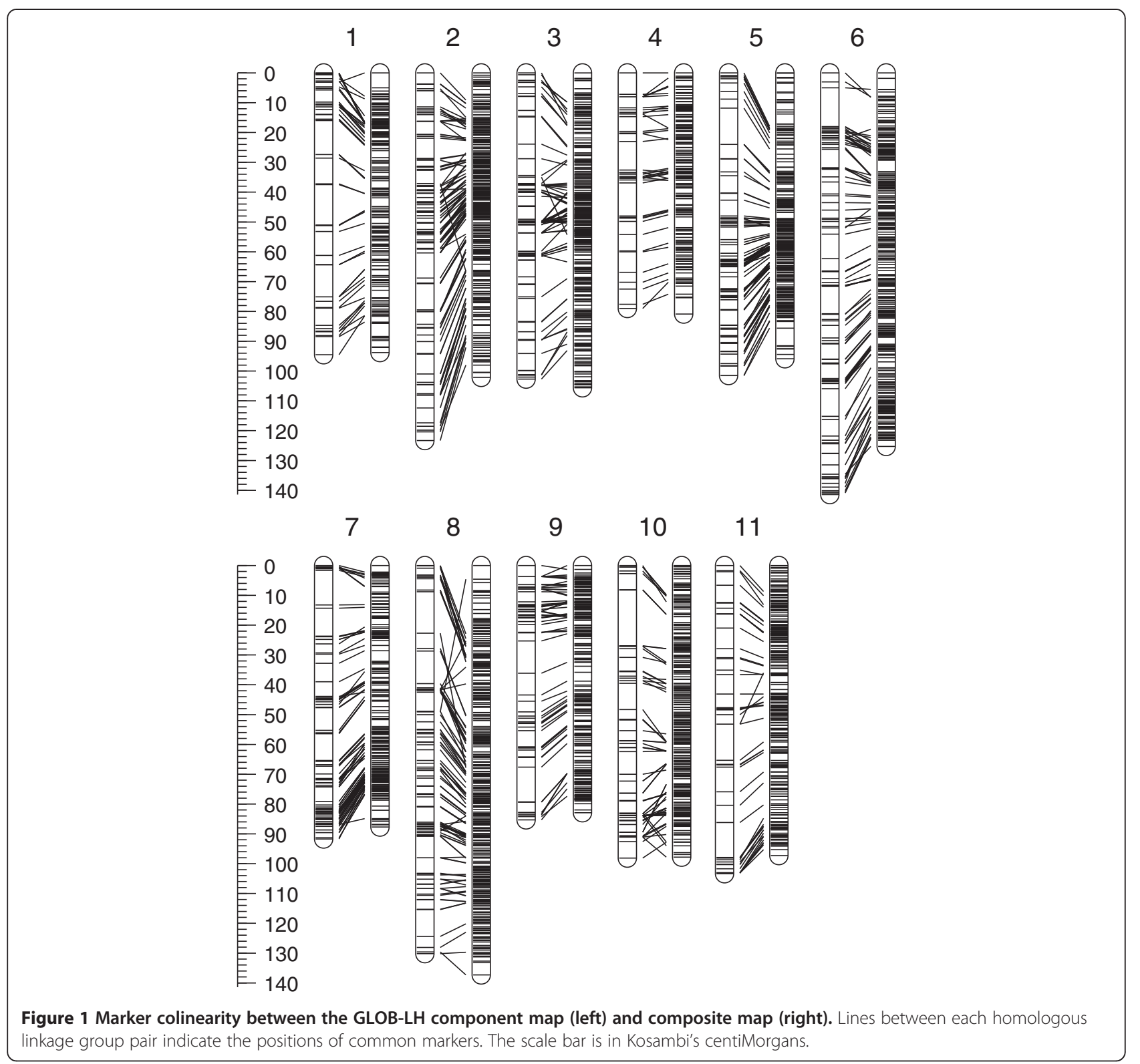

composite map and those component maps included in map construction ranged from 0.96 to 0.99 (Average $^{\text {b }}$ column; Table 3).

\section{DArT marker duplications}

Although not a main focus of this study, evidence for the occurrence of duplicated DArT marker loci within the assembled E. grandis genome sequence [36] was investigated for the five multicopy markers which had been mapped to three or more linkage groups. Two of these markers (ePt-637610 and ePt-637861; see Additional file 1) mapped to the same map position on each of four linkage groups (LGs 2, 3, 5 and 8) and were found to be redundant markers (i.e. identical sequences) based on their marker sequence similarity (bl2seq: 583/
606 base-pair similarity, e-value: 0.0). For the four unique multicopy markers, three were detected to have loci duplications within the E. grandis genome sequence. In each case, the positions of duplicated loci detected in the $E$. grandis genome sequence corresponded to the linkage groups to which the marker was mapped.

\section{Discussion}

\section{Composite map construction}

Data from seven component maps were integrated into a single composite map which represents the highest density map yet produced in Eucalyptus. A major advantage of the marker-merger method used in this study was the substantial time and labour savings made when compared to the effort required to produce comparable 
maps using traditional, segregation-based methods. For example, Li et al. [40] constructed a 2111 marker composite map from four barley mapping pedigrees and reported that it took 'several thousand hours' of computing time. In a larger barley study, Wenzl et al. [41] produced a 2935 loci composite map from ten mapping populations using JoinMap 3.0 [51] in combination with specially built Perl scripts and reported that the project required several months of semi-manual data processing [41]. In contrast, the composite map produced in this study was built in a single day.

\section{Utility of the composite map}

As sequences are available for the majority of DArT markers on the map (91\%; data not shown), the composite map provides a direct link to the E. grandis genome sequence [36]. We have made use of this link to search the E. grandis genome sequence for candidate genes associated with QTL locations and to facilitate the placement of candidate genes in the component linkage maps without the need for time consuming marker development and genotyping. Sequence-based linkage maps have also provided useful tools to aid in the assembly of genome sequences [e.g. 52,53] and can be particularly beneficial in taxa (such as eucalypts) which have a relatively small genome size. For example, during the assembly of the E. grandis genome sequence, a DArT linkage map was valuable in guiding contigs into the 11 main pseudochromosomes [16]. However, not all contigs could be aligned and approximately $12 \%$ of the $693 \mathrm{Mbp}$ E. grandis genome sequence remains unassembled in more than 4900 small unlinked scaffolds [54]. With the composite map containing many more DArT markers $(1600+)$ than the linkage map used to aid genome assembly, the composite map markers may provide further positional information and help to anchor some of the unlinked scaffolds and refine the current $E$. grandis genome sequence.

Over half (53\%) of the markers placed in the composite map originated from a single component map (i.e. were not shared among multiple component maps). Therefore, the ability to determine the relative positions of markers mapped in different maps has been greatly enhanced through the integration of this data into a single map. This has already proven advantageous to our research group, with the composite map being used to quickly identify the linkage relationships of microsatellite markers used in population genetic studies. Although now a relatively simple task, it was previously necessary to consult multiple linkage maps and assess their colinearity to obtain this same information. Furthermore, any marker developed in eucalypts which has known sequence, can now potentially be found in the eucalypt genome sequence and then aligned against the reference map in order to estimate its distance to other markers in units of recombination (cM); which are evolutionary meaningful units compared to base pair distances. Additionally, it is also important to understand the relationship between physical map (i.e. genome) and genetic map distances as this can have implications for map-based cloning efforts and/or marker-assisted selection. For example, uneven recombination rates across a genome $[12,55]$ may result in physically distant markers appearing to be genetically close to each other, or vice versa. In eucalypts, Kullan et al. [33] recently compared 153 linkage map intervals of approximately $1 \mathrm{cM}$ against contigs of the E. grandis genome and found that the genetic map to physical distance relationship varied considerably; ranging from $100 \mathrm{~kb}$ to $2.4 \mathrm{Mbp}$ per $1 \mathrm{cM}$. Therefore, the composite map will be useful to provide further insight into the relationship between physical and genetic map distance in addition to identifying hot (or cold) spots of recombination.

A key use of the composite map will be for comparison of QTL and candidate gene positions detected across variable genetic backgrounds and/or environments in different studies. This has previously been limited due to a lack of common markers being shared between maps [23]. For example, Thumma et al. [27] detected multiple co-locating growth-related QTL on LG5 in E. nitens but could not accurately compare the position of this QTL to similar growth-related trait QTL detected on this same linkage group in two other studies $[24,56]$. Although most of the markers contained on the composite map are DArT markers, which to date have only been mapped in the pedigrees included in this study, the map does contain several hundred microsatellite markers (213) which will enable synteny and colinearity to be established with many earlier linkage maps used for QTL detection; e.g. 13 out of 22 earlier studies have mapped a variable number of microsatellites [16]. This will enable QTL to be aligned against the composite map which may provide deeper insight into the genetic control of phenotypic traits in the genus. For example, following the construction of an integrated map for melon (Cucumis melo) which used data from eight independent mapping experiments, it was possible to align 370 QTL detected for 62 traits from 18 experiments [57]. Through this alignment, QTL detected in different studies for economically important traits were found to co-locate [57]; providing supporting evidence to substantiate the biological basis of the observed marker-trait association $[7,8]$.

As in all linkage mapping studies, it is important to consider both the quality of the map produced and any specific map characteristics. In the alignment of 6480 DArT marker sequences against the $E$. grandis genome sequence [36], Petroli et al. [11] reported that although the majority of markers (4189) occurred at a single genome position with high support, many marker sequences (2291), albeit at lower confidence, also exhibited similarity to a second 
genome position and that about half of these genome regions contained repeat elements. Furthermore, preliminary analysis of the E. grandis genome sequence suggests that (as has been observed in some Rosid genomes) a whole-genome duplication event has occurred in the lineage (Myrtales) subsequent to the ancient hexaploidy event shared by all rosids (Myburg et al., unpublished). Such whole-genome, as well as, segmental duplication events will affect thousands of marker loci, but most would be expected to diverge in sequence with evolutionary time yielding mostly unique marker loci. Thus, the presence of multicopy markers (representing putatively duplicated loci) in the composite map was not unexpected. It is worth noting that in the construction of each component map, only those markers which segregated as a single Mendelian locus were mapped. Therefore, in the event of a marker duplication being present within a pedigree, only one locus could be polymorphic in order for that marker to produce a single loci segregation ratios. Consequently, it is likely that only a subset of the duplicated loci present within the eucalypt genome have been identified in the composite map. Given that the PstI enzyme used in the complexity reduction step of DArT marker development [35] preferentially produces markers located in hypomethylated, gene rich regions [55], and that many DArT markers contain protein coding sequences [33], it is possible that some of the multicopy markers identified may be associated with different gene family members and/or be part of larger duplicated regions. Further studies are required to examine the full extent and evolution of the duplicated loci. We also expected some marker redundancy (markers with the same sequence) among the 3808 composite map DArT markers; an issue which arises due to the process by which DArT markers are generated, resulting in the same amplified genomic fragment being represented more than once on the genotyping array [31,35]. Therefore, identical clones (e.g. the same DArT fragment, but with different DArT marker names) are expected to produce identical genotype scores and should map to identical (or near identical) map positions; as found for the markers ePt637610 and ePt-637861 identified as identical clones in this study.

The marker-merging method used in this study took advantage of the fact that individual component maps were constructed using high marker-ordering stringency which resulted in linkage maps having robust marker-orders [32]. The comparison of the composite map marker-order against individual component maps gives an indication of the quality of the composite map. Marker-order correlations were mostly excellent with high pair-wise linkage group marker-order correlations found in most comparisons. For example, in 48 out of 66 pair-wise comparisons the marker-order correlation exceeded 0.95. Despite these high correlations, most component maps did exhibit some marker-order inconsistencies with the composite map. A number of (mostly) single marker-order inconsistencies did occur over large distances, but most marker-order disagreements occurred among tightly grouped markers in regions of less than $5 \mathrm{cM}$. Although it is possible that some of these marker-order differences could be real and represent local chromosomal rearrangements or marker duplications between the different mapping pedigrees and/or species, they are more likely to reflect markerorder inaccuracies within any of the component maps or simply be artefacts of the statistical uncertainty associated with ordering tightly linked markers [see 58]. While users of this map should be aware of these limitations and how they may affect marker ordering, overall, the generally high marker-order correlations observed and the exclusion of component map linkage groups having poor marker colinearity from initial composite map construction (and thus not adversely affecting composite map markerorder) suggests that the composite map is of a sufficiently high quality to facilitate the transfer of genetic information between studies.

The composite map will be most useful for studies involving species from subgenus Symphyomyrtus sections Latoangulatae and Maidenaria; due to the composite map being built from linkage maps constructed in species from these sections. However, due to the high level of genome synteny and colinearity detected between species from these relatively distant sections [28,32,34], information from the composite map should also be applicable to many other commercially important eucalypt species in closely related sections (e.g. E. camaldulensis from subgenus Symphyomyrtus section Exsertaria).

\section{Future marker integration}

A number of recent studies have focussed on the development of molecular markers for use in eucalypts. In addition to the DArT genotyping array developed for use in eucalypts [35], the feasibility of high-throughput SNP genotyping has been explored [59] and several tens of species-transferrable EST-based SSR markers have been recently reported $[60,61]$. Furthermore, DArT genotyping by sequencing (GBS), which combines the complexity reduction method of DArT [31] with next generation sequencing (NGS), and which can potentially deliver up to three-fold as many markers as conventional DArT genotyping [see 62] is becoming a cost-competitive genotyping option due to the recent plummeting costs of NGS sequencing. Therefore, to broaden the use of the composite map for comparative analyses and to optimise its' worth, it will be necessary to add new markers to the current version of the composite map in the future. Although beyond the scope of this study, it would also be valuable to compare the marker order of the composite map to maps built 
using the same data with other marker-merging software (e.g. BioMercator [63], CarthaGene [64] or MergeMap [65]). The R scripts and map marker positions of the component maps used in this study can be made available upon request.

\section{Conclusion}

The integration of markers from seven individual genetic linkage pedigrees has resulted in a composite, reference map for eucalypts with 4101 DArT and microsatellite markers. Although some small marker-order inconsistencies exist between component maps and the composite map, there is a relatively high agreement of markerorder between component maps; which indicates that the composite map represents a good estimation of the true marker positions in most cases. However, at finer scales (sub-cM) marker-orders may differ between component and composite maps due to limited statistical power to order such tightly linked markers. Overall, the genome coverage and marker density of the composite map greatly exceeded that achieved in any of the single mapping populations. It is expected that this composite map will provide a valuable reference map for the world-wide Eucalyptus research community, facilitate the transfer of genetic information between different studies and allow for the integration of DArT marker information with other genomic resources.

\section{Additional files}

\section{Additional file 1: Eucalyptus composite map details. Details of} markers mapped in the Eucalyptus composite map. Includes, linkage group and position of mapped markers, marker type and which component map(s) markers were mapped. A '1' in the 'Multicopy marker' column indicates that the marker occurs on two or more linkage groups.

Additional file 2: Composite map - component map marker colinearity. Marker colinearity among all six component maps and the Eucalyptus composite map. For each linkage group, three linkage group 'triplets' show marker colinearity between two component maps (outside) and the composite map (centre). Horizontal lines on linkage group bars indicate marker positions and lines between linkage groups indicate the position of common markers. The scale bar shown is in Kosambi's centiMorgans. Component map names (abbreviations; see Table 1) are given above each linkage group. Linkage groups excluded from composite map construction are indicated in parentheses following the component map name. An asterisk indicates whether marker-order information from the component map was incorporated during composite map construction (see Methods). For the GU-Emb component map, superscript letters indicates whether the framework ( $f$ ) or comprehensive (c) linkage group from this pedigree was used in composite map construction.

\section{Competing interest}

'The authors declare that they have no competing interests.

\section{Authors' contributions}

CJH built the GLOB-LH linkage map, coordinated the collection of map data, building of the composite map, performed analyses and wrote the manuscript. JSF built all other E. globulus linkage maps. AAM and ARKK, DG and CDP, built and contributed GU-SA and GU-Emb linkage maps, respectively. AK and FD constructed the composite map. REV and AAM conceived the study, and along with BMP and JSF, contributed to the design of the study. All authors read and approved the final manuscript.

\section{Funding}

Funding for this project was provided by the Australian Research Council (DP0770506 \& DP110101621) as well as the Cooperative Research Centre for Forestry (Australia). Construction of the GU-SA map was supported by Sappi, Mondi, the Technology and Human Resources for Industry Program (THRIP), the National Research Foundation (NRF) and the Department of Science and Technology (DST) of South Africa.

\section{Author details}

${ }^{1}$ School of Plant Science and CRC for Forestry, University of Tasmania, Private Bag 55 Hobart, Tasmania 7001, Australia. ${ }^{2}$ CRN Research Fellow, Faculty of Science, Health, Education and Engineering, University of the Sunshine Coast, Locked Bag 4, Maroochydore, QLD 4558, Australia. ³Department of Genetics, Forestry and Agricultural Biotechnology Institute (FABI), University of Pretoria, Pretoria 0002, South Africa. ${ }^{4}$ EMBRAPA Genetic Resources and Biotechnology - EPqB Final W5 Norte 70770-917 Brazilia DF and Dep. Cell Biology, Universidade de Brazilia - UnB, Brasilia, DF, Brazil. ${ }^{5}$ Diversity Arrays Technology Pty Ltd, PO Box 7141, Yarralumla, ACT 2600, Australia. ${ }^{6}$ EMBRAPA Genetic Resources and Biotechnology - Parque Estação Biológica - PqEB - Av. W5 Norte (final), Brasília, DF - Brazil - 70770-917, Universidade Catolica de Brasília- SGAN, 916 modulo B, 70790-160DF, Brasilia, Brazil.

Received: 23 March 2012 Accepted: 4 June 2012

Published: 15 June 2012

\section{References}

1. Krutovsky KV, Troggio M, Brown GR, Jermstad KD, Neale DB: Comparative mapping in the Pinaceae. Genetics 2004, 168:447-461.

2. Lefebvre-Pautigny F, Wu F, Philippot M, Rigoreau M, Zouine M, Frasse P, Bouzayen $M$, Broun $P$, Pétiard $V$, et al: High resolution synteny maps allowing direct comparisons between the coffee and tomato genomes. Tree Genetics \& Genomes 2009, 6:565-577.

3. Paterson AH, Bowers JE, Burow MD, Draye X, Elsik CG, Jiang C-X, Katsar CS, Lan T-H, Lin Y-R, Ming R, Wright RJ: Comparative genomics of plant chromosomes. Plant Cell 2000, 12:1523-1540.

4. Anderson JT, Lee C-R, Mitchell-Olds T: Life-history QTLs and natural selection on flowering time in Boechera stricta, a perennial relative of Arabidopsis. Evolution 2011, 65:771-787.

5. Freeman JS, O'Reilly-Wapstra JM, Vaillancourt RE, Wiggins N, Potts BM: Quantitative trait loci for key defensive compounds affecting herbivory of eucalypts in Australia. New Phytologist 2008, 178:846-851.

6. Kearsey MJ, Farquhar AGL: QTL analysis in plants; where are we now? Heredity 1998, 80:137-142.

7. Brown GR, Bassoni DL, Gill GP, Fontana JR, Wheeler NC, Megraw RA, Davis MF, Sewell MM, Tuskan GA, Neale DB: Identification of quantitative trait loci influencing wood property traits in loblolly pine (Pinus taeda L.). III. QTL verification and candidate gene mapping. Genetics 2003, 164:1537-1546.

8. Wheeler NC, Jermstad KD, Krutovsky K, Aitken SN, Howe GT, Krakowski J, Neale DB: Mapping of quantitative trait loci controlling adaptive traits in coastal Douglas-fir. IV. Cold-hardiness QTL verification and candidate gene mapping. Molecular Breeding 2005, 15:145-156.

9. Semagn K, Bjornstad A, Ndjiondjop MN: Principles, requirements and prospects of genetic mapping in plants. African Journal of Biotechnology 2006, 5:2569-2587.

10. The Potato Genome Sequencing Consortium: Genome sequence and analysis of the tuber crop potato. Nature 2011, 475:189-195.

11. Petroli C, Sansaloni C, Carling J, Mamani E, Steane D, Myburg A, Vaillancourt R, Kilian A, Pappas G, Bonfim da Silva O, Grattapaglia D: Genomic characterization, high-density mapping and anchoring of DArT markers to the reference genome of Eucalyptus. BMC Proceedings 2011, 5(Suppl 7):P35.

12. Jones $\mathrm{N}$, Ougham $\mathrm{H}$, Thomas $\mathrm{H}$, Pašakinskienè I: Markers and mapping revisited: finding your gene. New Phytologist 2009, 183:935-966.

13. Grattapaglia D, Plomion C, Kirst M, Sederoff RR: Genomics of growth traits in forest trees. Current Opinion in Plant Biology 2009, 12:148-156.

14. Poke F, Vaillancourt RE, Potts B, Reid J: Genomic research in Eucalyptus. Genetica 2005, 125:79-101. 
15. Grattapaglia D, Sederoff R: Genetic linkage maps of Eucalyptus grandis and Eucalyptus urophylla using a pseudo-testcross: mapping strategy and RAPD markers. Genetics 1994, 137:1121-1137.

16. Grattapaglia D, Vaillancourt RE, Shepherd M, Thumma BR, Foley W, Kulheim C, Potts BM, Myburg AA: Progress in Myrtaceae genomics: Eucalyptus as the pivotal genus. Tree Genetics \& Genomes, 8:463-508. in press.

17. Byrne M, Murrell JC, Allen B, Moran GF: An integrated genetic linkage map for eucalypts using RFLP, RAPD and isozyme markers. Theoretical and Applied Genetics 1995, 91:869-875.

18. Gan S, Shi J, Li M, Wu K, Wu J, Bai J: Moderate-density molecular maps of Eucalyptus urophylla (S. T. Blake) and E. tereticornis (Smith) genomes based on RAPD markers. Genetica 2003, 118:59-67.

19. Marques CM, Araújo JA, Ferreira JG, Whetten R, O'Malley DM, Liu BH, Sederoff R: AFLP genetic maps of Eucalyptus globulus and E. tereticornis. Theoretical and Applied Genetics 1998, 96:727-737.

20. Agrama HA, Salah SF: Construction of a genome map for Eucalyptus camaldulensis DEHN. Silvae Genetica 2002, 51:201-206.

21. Shepherd M, Kasem S, Lee D, Henry R: Construction of microsatellite linkage maps for Corymbia. Silvae Genetica 2006, 55:228-238.

22. Myburg AA, Potts BM, Marques CM, Kirst M, Gion J, Grattapaglia D, Grima-Pettenatti J: Eucalypts. In Genome mapping and molecular breeding in plants. Volume 7. Edited by Kole C. Berlin: Springer; 2007:115-160.

23. Brondani RPV, Williams ER, Brondani C, Grattapaglia D: A microsatellite-based consensus linkage map for species of Eucalyptus and a novel set of 230 microsatellite markers for the genus. BMC Plant Biology 2006, 6:20.

24. Freeman J, Whittock S, Potts B, Vaillancourt R: QTL influencing growth and wood properties in Eucalyptus globulus. Tree Genetics \& Genomes 2009, 5:713-722.

25. Gion J-M, Carouche A, Deweer S, Bedon F, Pichavant F, Charpentier J-P, Bailleres H, Rozenberg P, Carocha V, Ognouabi N, et al: Comprehensive genetic dissection of wood properties in a widely-grown tropical tree: Eucalyptus. BMC Genomics 2011, 12:301.

26. Marques C, Brondani R, Grattapaglia D, Sederoff R: Conservation and synteny of SSR loci and QTLs for vegetative propagation in four Eucalyptus species. Theoretical and Applied Genetics 2002, 105:474-478.

27. Thumma B, Baltunis B, Bell J, Emebiri L, Moran G, Southerton S: Quantitative trait locus (QTL) analysis of growth and vegetative propagation traits in Eucalyptus nitens full-sib families. Tree Genetics \& Genomes 2010, 6:877-889.

28. Myburg AA, Griffin AR, Sederoff RR, Whetten RW: Comparative genetic linkage maps of Eucalyptus grandis, Eucalyptus globulus and their $F_{1}$ hybrid based on a double pseudo-backcross mapping approach. Theoretical and Applied Genetics 2003, 107:1028-1042.

29. Appleby N, Edwards D, Batley J: New technologies for ultra-high throughput genotyping in plants. In Methods in Molecular Biology, Plant genomics. Volume 153. Edited by Somers DJ, Langridge P, Gustafson JP. New York: Humana Press; 2009:19-39.

30. Davey JW, Hohenlohe PA, Etter PD, Boone JQ, Catchen JM, Blaxter ML: Genome-wide genetic marker discovery and genotyping using next-generation sequencing. Nat Rev Genet 2011, 12:499-510.

31. Jaccoud D, Peng K, Feinstein D, Kilian A: Diversity arrays: a solid state technology for sequence information independent genotyping. Nucleic Acids Research 2001, 29(4):e25.

32. Hudson CJ, Kullan ARK, Freeman JS, Faria D, Grattapaglia D, Kilian A, Myburg A, Potts BM, Vaillancourt RE: High synteny and colinearity among Eucalyptus genomes revealed by high-density comparative genetic mapping. Tree Genetics \& Genomes 2012, 8:339-352.

33. Kullan A, van Dyk M, Jones N, Kanzler A, Bayley A, Myburg A: High-density genetic linkage maps with over 2,400 sequence-anchored DArT markers for genetic dissection in an $\mathrm{F}_{2}$ pseudo-backcross of Eucalyptus grandis $\mathrm{x}$ E. urophylla. Tree Genetics \& Genomes 2012, 8:163-175.

34. Steane DA, Nicolle D, Sansaloni CP, Petroli CD, Carling J, Kilian A, Myburg AA, Grattapaglia D, Vaillancourt RE: Population genetic analysis and phylogeny reconstruction in Eucalyptus (Myrtaceae) using high-throughput, genome-wide genotyping. Molecular Phylogenetics and Evolution 2011, 59:206-224.

35. Sansaloni C, Petroli C, Carling J, Hudson C, Steane D, Myburg A, Grattapaglia D, Vaillancourt R, Kilian A: A high-density Diversity Arrays Technology (DArT) microarray for genome-wide genotyping in Eucalyptus. Plant Methods 2010, 6:16.
36. Eucalyptus grandis genome (JG/ v1.0). http://www.phytozome.net/cgi-bin/ gbrowse/eucalyptus/.

37. Van Ooijen J: JoinMap 4, software for the calculation of genetic linkage maps in experimental populations. Wageningen, Netherlands: Kyazma B.V; 2006.

38. Grattapaglia D: Molecular breeding of Eucalyptus. In In Molecular biology of woody plants Volume 1. Edited by Jain S, Minocha S. Netherlands: Kluwer; 2000:451-474

39. Cheema J, Dicks J: Computational approaches and software tools for genetic linkage map estimation in plants. Briefings in Bioinformatics 2009, 10:595-608.

40. Li H, Kilian A, Zhou M, Wenzl P, Huttner E, Mendham N, McIntyre L, Vaillancourt R: Construction of a high-density composite map and comparative mapping of segregation distortion regions (SDRs) in barley. Molecular Genetics and Genomics 2010, 284:319-331.

41. Wenzl P, Li H, Carling J, Zhou M, Raman H, Paul E, Hearnden P, Maier C, Xia $L$, Caig V, et al: A high-density consensus map of barley linking DArT markers to SSR. RFLP and STS loci and agricultural traits. BMC Genomics 2006, 7:206.

42. Cone KC, McMullen MD, Bi IV, Davis GL, Yim Y-S, Gardiner JM, Polacco ML, Sanchez-Villeda H, Fang Z, Schroeder SG, et al: Genetic, physical, and informatics resources for Maize. On the road to an integrated map. Plant Physiology 2002, 130:1598-1605.

43. Mace E, Rami J-F, Bouchet S, Klein P, Klein R, Kilian A, Wenzl P, Xia L, Halloran $K$, Jordan D: A consensus genetic map of sorghum that integrates multiple component maps and high-throughput Diversity Array Technology (DArT) markers. BMC Plant Biology 2009, 9:13.

44. Alsop B, Farre A, Wenzl P, Wang J, Zhou M, Romagosa I, Kilian A, Steffenson B: Development of wild barley-derived DArT markers and their integration into a barley consensus map. Molecular Breeding 2011, 27:77-92.

45. Varshney R, Marcel T, Ramsay L, Russell J, Röder M, Stein N, Waugh R, Langridge $P$, Niks R, Graner A: A high density barley microsatellite consensus map with 775 SSR loci. Theoretical and Applied Genetics 2007, 114:1091-1103.

46. Wei F, Coe E, Nelson W, Bharti AK, Engler F, Butler E, Kim H, Goicoechea JL, Chen $M$, Lee $S$, et al: Physical and genetic structure of the Maize genome reflects its complex evolutionary history. PLoS Genet 2007, 3:e123.

47. Stam P: Construction of integrated genetic linkage maps by means of a new computer package: JoinMap. Plant J 1993, 3:739-744.

48. Bachir O, Abdellah B: Chromosome numbers of the 59 species of Eucalyptus L'Herit (Myrtaceae). Caryologia 2006, 59:207-212.

49. Voorrips RE: MapChart: software for the graphical presentation of linkage maps and QTLs. Journal of Heredity 2002, 93:77-78.

50. NCBI: NCBI BLAST. http://blast.ncbi.nlm.nih.gov/

51. Van Ooijen J, Voorrips RE: JoinMap 3.0, software for the calculation of genetic linkage maps. In Plant Research International. Wageningen, Netherlands; 2001

52. Wang Y, Sun S, Liu B, Wang H, Deng J, Liao Y, Wang Q, Cheng F, Wang X, Wu J: A sequence-based genetic linkage map as a reference for Brassica rapa pseudochromosome assembly. BMC Genomics 2011, 12:239.

53. Hwang T-Y, Sayama T, Takahashi M, Takada Y, Nakamoto $Y$, Funatsuki $H$, Hisano H, Sasamoto S, Sato S, Tabata S, et al: High-density integrated linkage map based on SSR markers in soybean. DNA Research 2009, 16:213-225.

54. Eucagen: Early release of the E. grandis genome sequence. http://web.up.ac.za/ eucagen/.

55. van Os H, Andrzejewski S, Bakker E, Barrena I, Bryan GJ, Caromel B, Ghareeb $B$, Isidore $E$, de Jong W, van Koert $P$, et al: Construction of a 10,000-marker ultradense genetic recombination map of potato: providing a framework for accelerated gene isolation and a genomewide physical map. Genetics 2006, 173:1075-1087.

56. Grattapaglia D, Bertolucci FLG, Penchel R, Sederoff RR: Genetic mapping of quantitative trait loci controlling growth and wood quality traits in Eucalyptus grandis using a maternal half-sib family and RAPD markers. Genetics 1996, 144:1205-1214.

57. Diaz A, Fergany M, Formisano G, Ziarsolo P, Blanca J, Fei Z, Staub J, Zalapa J, Cuevas $H$, Dace $G$, et al: A consensus linkage map for molecular markers and quantitative trait loci associated with economically important traits in melon (Cucumis melo L.). BMC Plant Biology 2011, 11:111.

58. Collard B, Mace E, McPhail M, Wenzl P, Cakir M, Fox G, Poulsen D, Jordan D: How accurate are the marker orders in crop linkage maps generated from large marker datasets? Crop and Pasture Science 2009, 60:362-372. 
59. Grattapaglia D, Silva-Junior O, Kirst M, de Lima B, Faria D, Pappas G: High-throughput SNP genotyping in the highly heterozygous genome of Eucalyptus: assay success, polymorphism and transferability across species. BMC Plant Biology 2011, 11:65.

60. Faria D, Mamani E, Pappas G, Grattapaglia D: Genotyping systems for Eucalyptus based on tetra-, penta-, and hexanucleotide repeat EST microsatellites and their use for individual fingerprinting and assignment tests. Tree Genetics \& Genomes 2011, 7:63-77.

61. Acuña C, Fernandez P, Villalba P, García M, Hopp H, Marcucci Poltri S: Discovery, validation, and in silico functional characterization of EST-SSR markers in Eucalyptus globulus. Tree Genetics \& Genomes 2012, 8:289-301.

62. Sansaloni $C P$, Petroli $C D$, Jaccoud D, Carling J, Detering F, Grattapaglia D, Kilian A: Diversity Arrays Technology (DArT) and next-generation sequencing combined: genome-wide, high throughput, highly informative genotyping for molecular breeding of Eucalyptus. BMC Proceedings 2011, 5(Suppl 7):P54.

63. Arcade A, Labourdette A, Falque M, Mangin B, Chardon F, Charcosset A, Joets J: BioMercator: integrating genetic maps and QTL towards discovery of candidate genes. Bioinformatics 2004, 20:2324-2326.

64. de Givry S, Bouchez M, Chabrier P, Milan D, Schiex T: CarthaGene: multipopulation integrated genetic and radiation hybrid mapping. Bioinformatics 2005, 21:1703-1704.

65. MergeMap. http://alumni.cs.ucr.edu/ yonghui/mgmap.html.

doi:10.1186/1471-2164-13-240

Cite this article as: Hudson et al:: A reference linkage map for Eucalyptus. BMC Genomics 2012 13:240.

\section{Submit your next manuscript to BioMed Central and take full advantage of:}

- Convenient online submission

- Thorough peer review

- No space constraints or color figure charges

- Immediate publication on acceptance

- Inclusion in PubMed, CAS, Scopus and Google Scholar

- Research which is freely available for redistribution 